${ }^{1}$ Faculté de Médecine, Université Paris-Sud, Université Paris-Saclay, Le Kremlin-Bicêtre, France. ${ }^{2}$ Service de Pneumologie, AP-HP, Le Kremlin-Bicetre, France. ${ }^{3}$ INSERM UMR_S999, Hôpital Marie Lannelongue, Le Plessis Robinson, France. ${ }^{4}$ Service de Pneumologie, CHU UcL Namur, Yvoir, Belgium. ${ }^{5}$ Sydney Medical School, University of Sydney, Camperdown, Australia. ${ }^{6}$ Département de Physiologie, AP-HP, Le Kremlin-Bicetre, France. ${ }^{7}$ Service de Pneumologie - Programme Hypertension Pulmonaire, Hôpitaux Universitaires de Genève, Geneva, Switzerland. ${ }^{8}$ Département de Chirurgie Thoracique, Vasculaire et de Transplantation Pulmonaire, Hôpital Marie Lannelongue, Le Plessis Robinson, France.

Correspondence: Edmund M. Lau, Department of Respiratory and Sleep Medicine, Royal Prince Alfred Hospital, University of Sydney, Missenden Rd, Camperdown NSW 2050, Australia. E-mail: edmund.lau@sydney.edu.au

Received: Oct 102015 | Accepted after revision: Feb 182016 | First published online: March 302016

Support statement: Funding for this manuscript was provided by Fondation Mont-Godinne.

Conflict of interest: Disclosures can be found alongside this article at erj.ersjournals.com

\title{
References
}

1 Hoeper MM, Bogaard HJ, Condliffe R, et al. Definitions and diagnosis of pulmonary hypertension. J Am Coll Cardiol 2013; 62: Suppl. 25, D42-D50.

2 Lau EMT, Humbert M, Celermajer DS. Early detection of pulmonary arterial hypertension. Nat Rev Cardiol 2014; 12: 143-155.

3 Naeije R, Vanderpool R, Dhakal BP, et al. Exercise-induced pulmonary hypertension: physiological basis and methodological concerns. Am J Respir Crit Care Med 2013; 187: 576-583.

4 Lewis GD, Bossone E, Naeije R, et al. Pulmonary vascular hemodynamic response to exercise in cardiopulmonary diseases. Circulation 2013; 128: 1470-1479.

5 Herve P, Lau EM, Sitbon O, et al. Criteria for diagnosis of exercise pulmonary hypertension. Eur Respir J 2015; 46: 728-737.

6 Kovacs G, Avian A, Olschewski H. Proposed new definition of exercise pulmonary hypertension decreases false positive cases. Eur Respir J 2016; 47: 1270-1273.

7 Portillo K, Torralba Y, Blanco I, et al. Pulmonary hemodynamic profile in chronic obstructive pulmonary disease. Int J Chron Obstruct Pulmon Dis 2015; 10: 1313-1320.

8 Kovacs G, Avian A, Tscherner M, et al. Characterization of patients with borderline pulmonary arterial pressure. Chest 2014; 146: 1486-1493.

9 Lau EM, Chemla D, Godinas L, et al. Loss of vascular distensibility during exercise is an early hemodynamic marker of pulmonary vascular disease. Chest 2016; 149: 353-361.

10 Naeije R, Chesler N. Pulmonary circulation at exercise. In: Terjung R, ed. Comprehensive Physiology. Hoboken, John Wiley \& Sons, Inc., 2012.

11 Tolle JJ, Waxman AB, Van Horn TL, et al. Exercise-induced pulmonary arterial hypertension. Circulation 2008 118: 2183-2189.

12 Kafi SA, Mélot C, Vachiéry JL, et al. Partitioning of pulmonary vascular resistance in primary pulmonary hypertension. J Am Coll Cardiol 1998; 31: 1372-1376.

\section{Multilobar bilateral and unilateral chest radiograph involvement: implications for prognosis in hospitalised community- acquired pneumonia}

To the Editor:

Community-acquired pneumonia (CAP) remains a leading cause of morbidity and mortality worldwide [1]. Studies from the USA and Europe suggest that severe CAP patients requiring admission to the intensive care unit (ICU) reach a mortality of up to $39 \%[2,3]$.

Since potential poor prognosis is known to contribute to increased ICU admissions, anticipating complications through the use of supporting measurements becomes essential. The 2007 Infectious Diseases Society of America (IDSA)/American Thoracic Society (ATS) guidelines [1] have redefined severe CAP and indications for ICU admission, with a rule consisting of major and minor clinical criteria. The rule is considered positive if one major or three minor criteria are present [1]. The presence of multilobar infiltrates is included among the minor criteria. However, multilobar pneumonia can be bilateral or unilateral and this 
difference may be of some importance. Our hypothesis was that radiographical bilateral pneumonia is an independent risk factor for mortality and that the prognosis for bilateral involvement is worse than that for multilobar unilateral involvement. We, therefore, studied the clinical characteristics and outcomes of bilateral pneumonia (at admission) compared to unilateral multilobar and localised pneumonia.

We performed a prospective observational study at Hospital Clínic, Barcelona, Spain. The study population consisted of adults with a diagnosis of CAP, consecutively examined from 2000 to 2013. In the initial visit, patients underwent a complete history and physical examination and laboratory testing. Patients were stratified into risk classes using the pneumonia severity index [4] and the CURB-65 scores (CURB-65: confusion, urea $>7 \mathrm{mmol} \cdot \mathrm{L}^{-1}$, respiratory rate $\geqslant 30$ breaths $\cdot \mathrm{min}^{-1}$, blood pressure $90 \mathrm{mmHg}$ (systolic) $\leqslant 60 \mathrm{mmHg}$ (diastolic), age $\geqslant 65$ years) [5]. All surviving patients were seen between 30 and 40 days after discharge. Multilobar pneumonia was defined as chest-radiograph infiltrates involving $\geqslant 2$ lobes; bilateral when the involved lobes were in both the right and left lungs, unilateral when the affected involved lobes were in the same lung, and localised when only a single pulmonary lobe was involved. Patients were categorised into three groups according to the presence of infiltrates: bilateral, multilobar unilateral and localised pneumonia. All CAP patients had lateral and anteroposterior (PA) projections to categorise radiographic involvement. All chest radiographs were reviewed by one specialist in lung radiology (M. Sánchez) to evaluate the radiographical pattern of infiltrate, number of lobes involved, and the presence of pleural effusion and atelectasis; the specialist was blinded to the clinical data.

Of the 5084 CAP patients screened, 4644 were included in this study. Of these, 1069 (23\%) had multilobar pneumonia and 585 (13\%) presented bilateral infiltrates; unilateral infiltrates were present in $484(45 \%)$ patients. Localised infiltrates were present in 3575 patients (77\%).

Patients from the bilateral group were younger, more frequently former alcohol consumers, had more often received previous antibiotic treatment, but less frequently influenza vaccine and inhaled corticosteroids, presented less frequently with chronic respiratory disease, chronic cardiovascular disease, or diabetes mellitus as a comorbidity, and pleuritic pain at admission. Patients from the unilateral multilobar group had a higher rate of chronic liver disease at admission. According to arterial oxygen tension $\left(\mathrm{PaO}_{2}\right)$ inspiratory oxygen fraction $\mathrm{FIO}_{2}$ ratio $\left(\mathrm{PaO}_{2} / \mathrm{FIO}_{2}\right)$ and the presence of infiltrates on the chest radiograph, we found significant differences between localised group versus unilateral multilobar group median (interquartile range) of 267 (226-314) versus 292 (252-335), $\mathrm{p}=0.001$ and between localised versus multilobar bilateral of 292 (252-335) versus 257 (219-300), $\mathrm{p}=0.001$. Interestingly we did not find differences between multilobar bilateral versus unilateral multilobar was 257 (219-300) versus 267 (226-314), $\mathrm{p}=0.28$.

Confirmed aetiology was found in 1821 (39\%) patients. The most frequent pathogens were Streptococcus pneumoniae ( $\mathrm{n}=768,42 \%)$. Pathogens did not differ between groups, except for $S$. pneumoniae, which was less frequent in bilateral patients (bilateral 34\%, unilateral multilobar 44\%, and localised 43\%; $\mathrm{p}=0.013$ ), and Staphylococcus aureus was more frequent in the unilateral multilobar group (bilateral 3\%, unilateral multilobar 5\% and localised 2\%; $\mathrm{p}=0.025)$.

Of the 585 patients with bilateral involvement, 198 (34\%) were admitted to the ICU; 81 (16\%) patients required invasive mechanical ventilation more frequently than the unilateral multilobar $(n=39,10 \%)$ and localised groups $(n=97,3 \%),(p<0.001)$. Patients with bilateral involvement had a longer stay in hospital than the localised group (bilateral 8 days; unilateral multilobar 9 days and localised 6 days; $\mathrm{p}<0.001$ ). A total of $260(6 \%)$ patients died and a 30-day mortality was significantly higher among bilateral patients (bilateral 11\%, unilateral multilobar $7 \%$ and localised $4 \%$; $<<0.001$ ).

In the multivariate analysis, age $(\geqslant 65$ years), neurological disease, chronic liver disease, altered mental status, $\mathrm{PaO}_{2} / \mathrm{FIO}_{2}<250$, acute renal failure, septic shock, interstitial pattern, and bilateral involvement were risk factors for 30-day mortality. Unilateral multilobar involvement was not an independent factor associated with 30-day mortality (table 1). The area under the receiver operating characteristic (ROC) curve of the predictive model was 0.88 (95\% CI 0.85-0.90).

In a large population of CAP patients, we showed that $23 \%$ of patients with CAP had multilobar infiltrates and $13 \%$ showed bilateral multilobar involvement on the chest radiograph. Our main findings are: 1) the clinical course of the bilateral group was severe, with more patients requiring admission to the ICU and mechanical ventilation compared to patients with unilateral multilobar and localised disease; and 2) more importantly, the presence of bilateral involvement was an independent predictive factor for mortality, while unilateral multilobar disease was not.

The association of multilobar involvement with prognosis has been previously investigated in some studies [6-8], as it is one of the IDSA/ATS minor criteria for ICU admission in CAP [1]. Many studies have evaluated these criteria, especially the minor criteria $[6,7,9-12]$, with conflicting results. In the study by Chalmers et al. [10], the predictive value of multilobar shadowing was strong, with an OR of 4.20 
TABLE 1 Significant univariate and multivariate logistic regression analyses of predictors for 30-day mortality

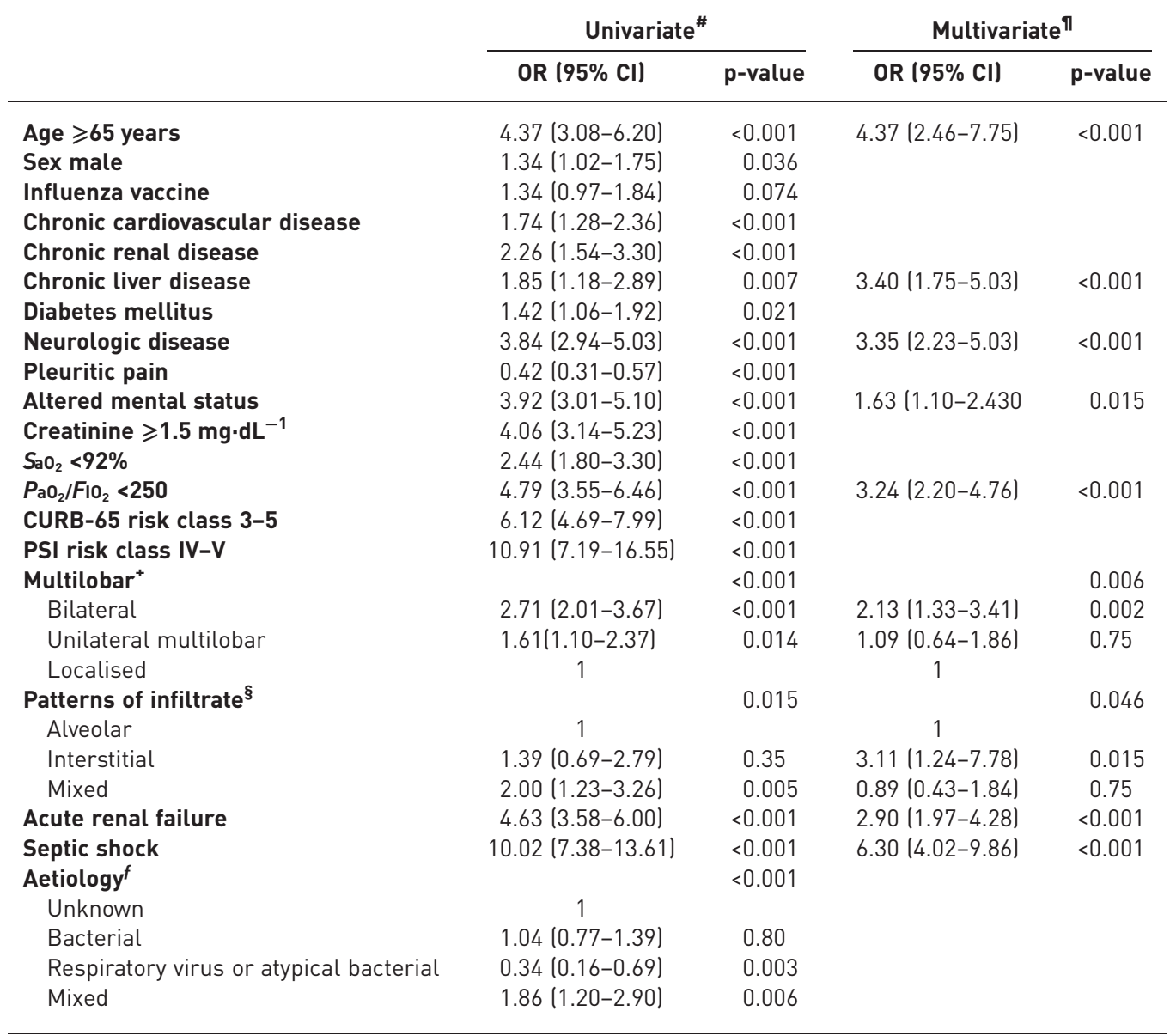

$\mathrm{SaO}_{2}$ : arterial oxygen saturation; $\mathrm{PaO}_{2}$ : arterial oxygen tension; $\mathrm{FlO}_{2}$ : inspiratory oxygen fraction; CURB-65: confusion, urea $>7 \mathrm{mmol} \cdot \mathrm{L}^{-1}$, respiratory rate $\geqslant 30$ breaths $\cdot \mathrm{min}^{-1}$, blood pressure, $90 \mathrm{mmHg}$ (systolic) $\leqslant 60 \mathrm{mmHg}$ (diastolic), age $\geqslant 65$ years; PSI: pneumonia severity index. \#: the variables included in the univariate analysis were age, sex, smoking, alcohol consumption, previous antibiotic, influenza vaccine, pneumococcal vaccine, inhaled corticosteroids, systemic corticosteroids, chronic pulmonary disease, chronic cardiovascular disease, diabetes mellitus, neurological disease, chronic liver disease, pleuritic pain, altered mental status, creatinine, C-reactive protein level, white blood cell count, $\mathrm{SaO}_{2}, \mathrm{PaO}_{2} / \mathrm{FlO}_{2}, \mathrm{CURB}-65$ risk class, PSI risk class, multilobar, pattern of infiltrate, septic shock, acute renal failure, and aetiology. ": HosmerLemeshow goodness-of-fit test, $p=0.17$. Internal validation of the final logistic regression model was conducted using bootstrapping with 1000 samples. The nine variables included in the model showed robust results, with small $95 \% \mathrm{Cls}$ around the original coefficients. ${ }^{+}$: the p-value corresponds to the differences between the three groups (bilateral, unilateral multilobar, or localised). ${ }^{\S}$ : the $p$-value corresponds to the differences between the three groups (alveolar, interstitial, or mixed). ${ }^{f}$ : the $p$-value corresponds to the differences between the four groups (unknown, bacterial, respiratory virus or atypical bacterial, or mixed).

(95\% CI 2.56-6.88) for mechanical ventilation/vasopressor support (MV/VS) and an OR of 5.63 (95\% Cl 3.09-10.3) for 30-day mortality.

AliberTi et al. [13] in a multicentre observational study of CAP cases observed the highest in-hospital mortality among CAP patients with acute respiratory failure, severe sepsis and multilobar infiltrates (26\%).

RELLO et al. [14], in a study evaluating 428 ICU patients with CAP, including 126 chronic obstructive pulmonary disease (COPD) patients, reported that ICU mortality in COPD patients with adequate therapy was associated with bilateral infiltrates and shock. In fact, their study and that of WALDEN et al. [15], which included patients with severe CAP, looked at bilateral involvement as a predictor of mortality.

Similarly, the meta-analysis by MANNU et al. [16] has shown multilobar involvement to be associated with unfavourable outcomes, such as a significantly increased risk of mortality (OR 2.57, 95\% CI 1.83-3.61) in all seven studies analysed. Bilateral involvement was not analysed separately. 
We also examined the mortality factors in the group of patients with bilateral and unilateral multilobar pneumonia and found that bilateral, but not unilateral involvement was an independent predictive factor for 30-day mortality in CAP patients.

The strengths of this study are the large number of patients enrolled over a long period of time, its prospective design, and the comprehensive clinical and microbiological data gathered. Limitations include the fact that it is a single-centre study, therefore the results need to be validated in external cohorts, and the lack of computed tomography (CT) scan data to compare chest radiographs with. Recently, ClaESSENS et al.[17] highlighted specificity and sensitivity problems of chest radiographs compared to CT scans in CAP. However, performing CT scans for CAP is unfeasible in routine clinical practice.

In summary, bilateral chest radiographs involvement was an independent factor associated with higher mortality, whereas unilateral multilobar pneumonia was not. We suggest including bilateral instead of multilobar pneumonia in the scores for prognosis and ICU admission in CAP.

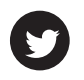

@ERSpublications

Bilateral but not unilateral multilobar pneumonia is an independent risk factor for mortality in CAP http://ow.ly/ZNLD4

\begin{abstract}
Adamantia Liapikou', Catia Cillóniz ${ }^{2}$, Albert Gabarrús², Rosanel Amaro², Jorge Puig De la Bellacasa ${ }^{3}$, Josep Mensa ${ }^{4}$, Marcelo Sánchez ${ }^{5}$, Michael Niederman ${ }^{6}$ and Antoni Torres ${ }^{2}$

${ }^{1}$ 6th Respiratory Dept, Sotiria Hospital, Athens, Greece. ${ }^{2}$ Dept of Pneumology, Institut Clinic del Tórax, Hospital Clínic of Barcelona - Institut d'Investigacions Biomèdiques August Pi i Sunyer (IDIBAPS), University of Barcelona (UB) - SGR 911 - Ciber de Enfermedades Respiratorias (Ciberes) Barcelona, Spain. ${ }^{3}$ Microbiology Dept, Hospital Clínic, Barcelona, Spain. ${ }^{4}$ Infectious Diseases Dept, Hospital Clínic, Barcelona, Spain. ${ }^{5}$ Radiology Department, Hospital Clínic, Barcelona, Spain. ${ }^{6}$ Pulmonary and Critical Care Division, Weill Cornell Medical College, New York, NY, USA.
\end{abstract}

Correspondence: Antoni Torres, Dept of Pneumology, Hospital Clínic of Barcelona, C/ Villarroel 170, 08036 Barcelona, Spain. E-mail: atorres@clinic.ub.es

Received: Jan 262016 | Accepted after revision: March 082016 | First published online: April 212016

Support statement: This work was supported by Ciber de Enfermedades Respiratorias (CibeRes CB06/06/0028). 2009 Support to Research Groups of Catalonia 911.

Conflict of interest: None declared.

Acknowledgements: We are indebted to all medical and nursing colleagues for their assistance and cooperation in this study.

\title{
References
}

1 Mandell LA, Wunderink RG, Anzueto A, et al. Infectious Diseases Society of America/American Thoracic Society consensus guidelines on the management of community-acquired pneumonia in adults. Clin Infect Dis 2007; 44: Suppl. 2, S27-S72.

2 Lim WS, Baudouin SV, George RC, et al. BTS guidelines for the management of community acquired pneumonia in adults: update 2009. Thorax 2009; 64: Suppl. 3, iiil-iii55.

3 Garcia-Vidal C, Fernández-Sabe N, Carratala J, et al. Early mortality in patients with community-acquired pneumonia: causes and risk factors. Eur Respir J 2008; 32: 733-739.

4 Fine MJ, Auble TE, Yealy DM, et al. A prediction rule to identify low-risk patients with community-acquired pneumonia. N Engl J Med 1997; 336: 243-250.

5 Lim WS, van der Eerden MM, Laing R, et al. Defining community acquired pneumonia severity on presentation to hospital: an international derivation and validation study. Thorax 2003; 58: 377-382.

6 Liapikou A, Ferrer M, Polverino E, et al. Severe community-acquired pneumonia: validation of the Infectious Diseases Society of America/American Thoracic Society Guidelines to predict an intensive care unit admission. Clin Infect Dis 2009; 48: 377-385.

7 Chalmers JD, Taylor JK, Mandal P, et al. Validation of the Infectious Diseases Society of America/American Thoratic Society minor criteria for intensive care unit admission in community-acquired pneumonia patients without major criteria or contraindications to intensive care unit care. Clin Infect Dis 2011; 53: 503-511.

8 Lim HF, Phua J, Mukhopadhyay A, et al. IDSA/ATS minor criteria aid pre-intensive care unit resuscitation in severe community-acquired pneumonia. Eur Respir J 2014; 43: 852-862.

9 Viasus D, Garcia-Vidal C, Manresa F, et al. Risk stratification and prognosis of acute cardiac events in hospitalized adults with community-acquired pneumonia. J Infect 2013; 66: 27-33.

10 Chalmers JD. ICU admission and severity assessment in community-acquired pneumonia. Crit Care 2009; 13: 156.

11 Phua J, See KC, Chan YH, et al. Validation and clinical implications of the IDSA/ATS minor criteria for severe community-acquired pneumonia. Thorax 2009; 64: 598-603.

12 Singanayagam A, Chalmers JD, Hill AT. Severity assessment in community-acquired pneumonia: a review. QJM 2009; 102: 379-388.

13 Aliberti S, Brambilla AM, Chalmers JD, et al. Phenotyping community-acquired pneumonia according to the presence of acute respiratory failure and severe sepsis. Respir Res 2014; 15: 27.

14 Rello J, Rodriguez A, Torres A, et al. Implications of COPD in patients admitted to the intensive care unit by community-acquired pneumonia. Eur Respir J 2006; 27: 1210-1216. 
15 Walden AP, Clarke GM, McKechnie S, et al. Patients with community acquired pneumonia admitted to European intensive care units: an epidemiological survey of the GenOSept cohort. Crit Care 2014; 18: R58.

16 Mannu GS, Loke YK, Curtain JP, et al. Prognosis of multi-lobar pneumonia in community-acquired pneumonia: a systematic review and meta-analysis. Eur J Intern Med 2013; 24: 857-863.

17 Claessens YE, Debray MP, Tubach F, et al. Early chest computed tomography scan to assist diagnosis and guide treatment decision for suspected community-acquired pneumonia. Am J Respir Crit Care Med 2015; 192: 974-982.

\title{
Implementing tuberculosis entry screening for asylum seekers: the Groningen experience
}

\author{
To the Editor:
}

For 3 years, Europe has faced an increasing refugee crisis in which hundreds of thousands of displaced persons are risking their lives to seek a safer and better future in the European Union (EU). Many of these migrants originate from highly tuberculosis (TB)-endemic countries. Furthermore, their travel conditions are often poor, allowing potential transmission of infectious diseases, including TB. The Netherlands have observed an increased influx of asylum seekers since 2012 too, with fluctuating numbers every month, and a sharp increase in the summer of 2015, putting constraints on the process of registration, identification, application and accommodation, including mandatory radiographic screening for intrathoracic TB. We describe the impact of such migration on the Public Health TB Clinic of Groningen (responsible for nearly all TB entry screening for asylum seekers in the Netherlands), and our flexible and efficient practice model for daily radiographic TB screening. We report the yield of this intervention over the last 4 years, and reflect on issues concerning entry screening.

In the Netherlands, a centralised system of asylum application is in operation. Apart from a small minority at the national airport, Schiphol (Amsterdam), and unaccompanied minors, the majority of asylum seekers must file their request at the national reception centre in Ter Apel, a former military North Atlantic Treaty Organization base, where they are received under basic conditions in prefabricated units in one of the hangars. The procedure of 1) registration and identity verification by the Alien Police, 2) mandatory radiographic screening for intrathoracic $\mathrm{TB}$, and 3) an interview with the Immigration and Naturalisation Service must be completed within a time frame of $72 \mathrm{~h}$.

Since April 2012, TB screening has been performed on a daily basis, including weekends, with a mobile digital X-ray unit parked in another hangar. Because of the increasing influx in 2014 and 2015, two annex reception centres were opened in the towns of Veenhuizen and Budel, the first one also being served by the Public Health TB Clinic of Groningen. Chest radiographs are immediately digitally transferred for reading to the Public Health TB Clinic in Groningen, approximately $50 \mathrm{~km}$ away, allowing for teleradiology. The chest radiographs from Ter Apel are read on a daily basis by one of the two public health TB physicians working in the Public Health TB Clinic. The chest radiographs from Veenhuizen are read by one of the two consultant chest physicians working in the modern TB sanatorium Beatrixoord (part of the University Medical Center Groningen (UMCG)). This is one of two national tertiary referral and expertise TB centres in the Netherlands. On Saturdays and public holidays, a group of Dutch TB physicians reads the chest radiographs on a rotational basis.

At the national reception centre, the Public Health TB Clinic uses a caravan as an on-site basic TB clinic. Apart from making chest radiographs, upon request of the TB physician or chest physician, the team of medical technical assistants from the Public Health TB Clinic in Groningen can, daily, on location, 1) take standardised questionnaires with telephone translators, 2) collect sputum for bacteriology, 3) perform tuberculin skin testing or 4) isolate individuals with abnormal chest radiographs possibly compatible with active intrapulmonary TB in three other caravans (with any relatives), awaiting the results of sputum-smear microscopy and GeneXpert (Cepheid, Sunnyvale, CA, USA) examination. Individuals with a chest radiograph highly suggestive of infectious pulmonary TB may be referred directly to Beatrixoord, which is equipped with state-of-the-art isolation rooms for 20 persons in addition to 10 beds for noninfectious TB patients, where further isolation, examination and treatment is offered. In the absence of productive cough, a bronchoscopy is arranged in the Chest Clinic of the UMCG. Sputum and bronchial lavage smear-microscopy and direct PCR test for Mycobacterium tuberculosis 